\title{
Melanoma Maligno Cutâneo: Estudo Retrospetivo de Sete Anos (2011-2017)
}

\author{
Filipa Tavares Almeida', R. Caldas', P. Oliveira², Celeste Brito' \\ 'Serviço de Dermatovenereologia, Hospital de Braga, Braga, Portugal \\ ${ }^{2}$ Departamento de Estudo de Populações, Instituto de Ciências Biomédicas Abel Salazar, Universidade do Porto, Porto, Portugal
}

RESUMO - Introdução: O melanoma maligno é uma neoplasia cutânea muito agressiva, com uma incidência crescente nos últimos anos. Material e Métodos: Realizou-se estudo retrospetivo através da análise de processos clínicos de doentes com o diagnóstico de melanoma maligno cutâneo, durante o período compreendido entre 2011 e 2017. Analisaram-se características epidemiológicas, clínicas e histopatológicas e comparou-se com um estudo prévio de 11 anos realizado nesta Instituição (1999 a 2009). Resultados: O melanoma maligno cutâneo foi diagnosticado em 163 doentes, 59,5\% do sexo feminino e 40,5\% do sexo masculino, cuja idade média foi de 65,6 anos. A localização anatómica mais frequente nos homens foi o tronco, enquanto nas mulheres foi a perna. O subtipo de melanoma maligno cutâneo mais comum foi o de extensão superficial, seguindo-se o lentigo maligno e o nodular. A maioria dos casos eram melonoma maligno cutâneo invasivos (67,5\%), em oposição aos melanomas malignos cutâneos in situ (32,5\%). A média do índice de Breslow foi de 3,01 mm. Destaca-se o aumento do número de doentes com melanoma maligno cutâneo, já que no estudo anterior apenas tinham sido diagnosticados 129 casos. Conclusão: Os resultados obtidos neste estudo estão de acordo com o descrito na literatura, nomeadamente no que se refere à faixa etária dos doentes, e à localização anatómica e subtipo predominante do melanoma maligno cutâneo. Verificou-se um aumento do número de casos localizados na cabeça e tronco face aos membros inferiores. Apesar do investimento na deteção do melanoma maligno cutâneo, o aumento do número de diagnósticos não parece contrabalançar o risco global de melanoma maligno cutâneo, uma vez que continuam a surgir muitos casos, predominantemente invasivos e espessos.

PALAVRAS-CHAVE - Melanoma; Neoplasias da Pele.

\section{Cutaneous Malignant Melanoma: A 7-Year Retrospective Study (2011-2017)}

\begin{abstract}
Introduction: Malignant melanoma is a very aggressive cutaneous neoplasm, with an increasing incidence in the last years. Material and Methods: A retrospective study was conducted through the analysis of patient's files diagnosed with cutaneous malignant melanoma between 2011 and 2017. Epidemiological, clinical and histopathological characteristics were analyzed and compared with a previous study from this institution (1999 to 2009). Results: Cutaneous malignant melanoma was diagnosed in 163 patients, 59.5\% female and 40.5\% male, with a mean age of 65.6 years. The most frequent anatomical location in men was the trunk, while in women it was the leg. The most common subtype of cutaneous malignant melanoma was the superficial spreading, followed by the lentigo maligna and the nodular. The majority of the cases were invasive cutaneous malignant melanoma (67.5\%) in opposition to cutaneous malignant melanoma in situ (32.5\%). The mean Breslow index was $3.01 \mathrm{~mm}$. It is important to note the increase in the patient's number with cutaneous malignant melanoma, given that in the previous study only 129 cases had been diagnosed. Conclusion: Our data are in agreement with literature, regarding the patient's age group, and the predominant anatomic location and subtype of cutaneous malignant melanoma. There was an increase in the number of cases located in the head and trunk compared to the lower limbs. Despite the efforts to detect cutaneous malignant melanoma, earlier diagnosis could not counterbalance the global risk of cutaneous malignant melanoma, as we still observe many cases, predominantly invasive and thick.
\end{abstract}

KEYWORDS - Melanoma; Skin Neoplasms.

Correspondência: Bruno Duarte

Filipa Tavares Almeida

Rua Sete Fontes - 4710-243 Braga

E-mail: filipa.almeida6@hotmail.com

DOI: https://dx.doi.org/10.29021/spdv.77.2.1065
Recebido/Received

18 Abril/April 2019

Aceite/Accepted

1 Maio/May 2019 


\section{Artigo Original}

\section{INTRODUÇÃO}

O melanoma maligno é uma neoplasia cutânea muito agressiva, sendo responsável por $75 \%$ das mortes atribuídas a todas as neoplasias cutâneas. ' Segundo a European Network of Cancer Registries, em 2008 foram estimadas mais de 20 mil mortes por melanoma maligno na Europa. ${ }^{2}$

A sua incidência tem aumentado de forma rápida e consistente ao longo dos últimos 30 anos, particularmente na população caucasiana e indivíduos com fotótipos baixos. Estima-se que este aumento seja de $3 \%$ a $7 \%$ por ano, resultando numa duplicação da incidência global do melanoma maligno cutâneo (MMC) a cada 15 a 20 anos. ${ }^{3}$ Acresce que na última década houve uma estabilização do aumento da incidência na população com idade inferior a 50 anos. ${ }^{4}$

Assim, é fundamental o conhecimento das características epidemiológicas, clínicas e histopatológicas do melanoma maligno, tendo em vista a implementação de programas de prevenção primária e o aperfeiçoamento da sua abordagem, favorecendo, em particular, o diagnóstico precoce com consequente diminuição da mortalidade.

Este estudo teve como objetivo caracterizar os casos de melanoma maligno cutâneo diagnosticados no serviço de Dermatovenereologia do Hospital de Braga durante um período de 7 anos.

\section{MATERIAL E MÉTODOS}

Foi realizado um estudo retrospetivo no Serviço de Dermatovenereologia do Hospital de Braga, através da análise de processos clínicos de doentes com o diagnóstico de $M M C$, durante o período compreendido entre janeiro de 2011 e dezembro de 2017. Neste trabalho analisaram-se características epidemiológicas, clínicas e histopatológicas e comparou-se com um estudo anterior realizado nesta Instituição. A análise estatística descritiva foi efetuada através do programa SPSS, versão 25.

\section{RESULTADOS}

Foram identificados 163 doentes, 97 do sexo feminino $(59,5 \%)$ e 66 do sexo masculino $(40,5 \%)$. A média de idade no momento do diagnóstico foi de 65,6 e a mediana de 70 (1599 anos). Quando analisado por género, a idade média de diagnóstico nas mulheres foi de 64 anos e nos homens de 68 anos. A maioria dos doentes tinha mais de 50 anos $(78,5 \%)$. Menos de $10 \%$ dos doentes tinham idade inferior a 40 anos.

Durante o período de estudo, o número de casos de MMC oscilou, com uma média de 23 casos/ano. Não se observaram diferenças estatisticamente significativas no que toca à incidência anual do $M M C$, inclusivamente quando analisado por género e faixa etária $(p>0,05)$.

O tronco foi identificado como a localização prevalente de $M M C$, seguindo-se a cabeça e perna (Fig. 1). No entanto, quando realizada distribuição por género, no sexo feminino verificou-se um predomínio de $M M C$ na cabeça/pescoço e perna. No sexo masculino, o tronco mantém-se como a localização mais frequente (Fig. 2). Importa referir que nos casos da cabeça e pescoço a idade média ao diagnóstico foi

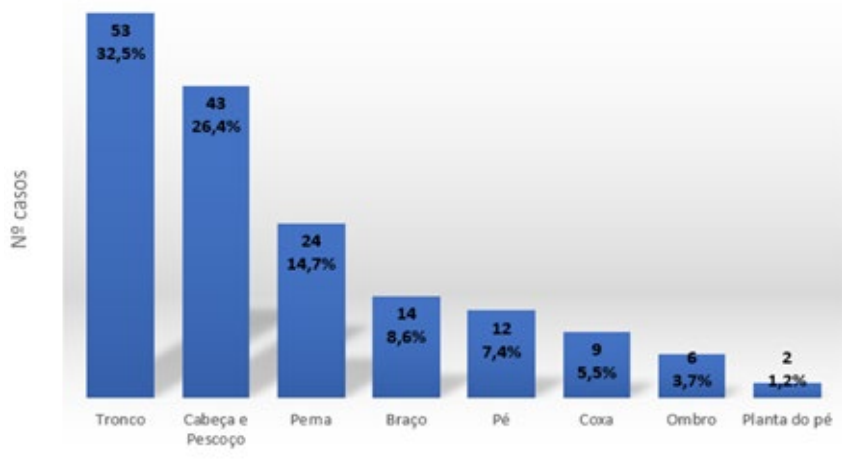

Figura 1 - Localização anatómica dos casos de MMC.

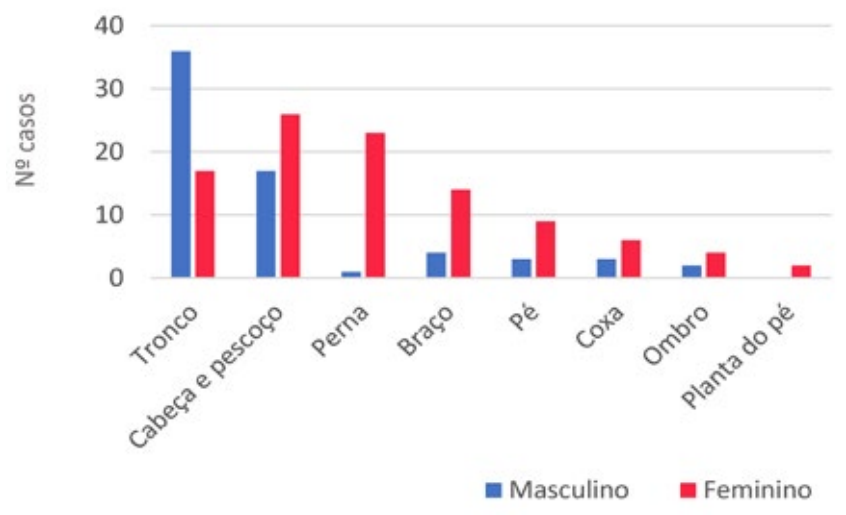

Figura 2 - Localização anatómica dos casos de MMC, distribuída por género.

superior aos de diferente localização anatómica $(76,4$ anos em comparação com 62,0 anos).

Histologicamente, o subtipo de extensão superficial representou $55,8 \%$ dos casos. O segundo mais frequente foi o lentigo maligno $(19,6 \%)$, seguindo-se o nodular $(18,4 \%)$ e acral lentiginoso $(4,9 \%)$.

No tronco verificou-se um predomínio do subtipo de extensão superficial $(77,4 \% ; 41 / 53)$, e na cabeça e pescoço predominou o lentigo maligno $(69,8 \% ; 30 / 43)$.

A análise histopatológica revelou que a maioria das lesões diagnosticadas foram MMC invasivos $167,5 \%$; $110 / 163)$, enquanto que os $M M C$ in situ ocorreram em $32,5 \%$ dos casos (53/163). Nestes últimos, a idade média ao diagnóstico foi superior à dos $M M C$ invasivos $(71,3$ anos em comparação com 62,9 anos). A localização anatómica prevalente nos $M M C$ in situ foi a cabeça e pescoço $(62,3 \%$; $33 / 53)$, seguindo-se o tronco $(13,2 \% ; 7 / 53)$.

No que concerne aos MMC invasivos, o índice de Breslow variou entre 0,04 mm e $19 \mathrm{~mm}$ (média 3,01 \pm 3,59; mediana 1,29). Apesar de haver um predomínio dos $M M C$ considerados finos (índice de Breslow $\leq 1 \mathrm{~mm}$ ), 23,6\% dos casos tinham espessura superior a $4 \mathrm{~mm}$. Prevaleceu 
a localização no tronco $(43,6 \% ; 48 / 110)$ e perna $(21,8 \%$; $24 / 110)$, seguindo-se o braço e o pé, ambos descritos em $8,2 \%$ dos casos (9/110).

A maior espessura média do tumor foi observada na cabeça e região acral e a menor no tórax, correspondendo ao subtipo de extensão superficial.

Observou-se uma incidência crescente dos MMC invasivos, principalmente à custa dos espessos, em comparação com os MMC in situ embora não se tenha verificado diferença estatisticamente significativa $(p>0,05)$ (Fig. 3).

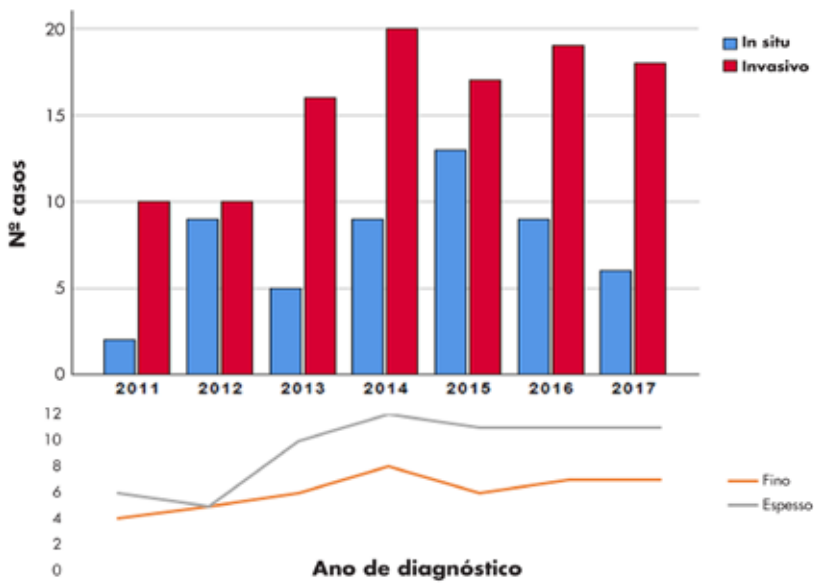

Figura 3 - Distribuição dos casos de MMC por ano de diagnóstico e espessura tumoral.

\section{DISCUSSÃO}

Os resultados obtidos neste estudo estão de acordo com a literatura e também com o estudo prévio, relativamente ao predomínio do sexo feminino, faixa etária dos doentes, localização anatómica e subtipo histológico de MMC. ${ }^{5-7}$

Cerca de $80 \%$ doentes tinha idade superior a 50 anos no momento do diagnóstico e é expectável que a média de idades venha a aumentar, a par do aumento da esperança média de vida. ${ }^{8}$

Classicamente existe uma predominância do MMC na perna em mulheres e no tronco em homens, geralmente atribuídas a variações de comportamento entre os géneros, por motivos de profissão, vestuário e exposição ao sol, que parecem estar a diminuir gradualmente. ${ }^{9}$ Dada a mudança de padrões comportamentais, a cabeça é atualmente uma das localizações anatómicas mais observada no sexo feminino. ${ }^{10}$ Para além disso, houve uma diminuição dos casos localizados nas pernas, coincidindo com o aumento de casos na cabeça e tronco. ${ }^{11}$

$\mathrm{Na}$ verdade, como observado no nosso estudo, os MMC da cabeça e pescoço têm vindo a aumentar significativamente e o diagnóstico tende a ocorrer em idades mais avançadas. ${ }^{22} \mathrm{O}$ facto do maior número de casos corresponder a MMC in situ está de acordo com o subtipo histológico mais frequente ser o lentigo maligno. ${ }^{13}$ Assim, perante o aumento da esperança média de vida, estima-se que, num futuro próximo, estes casos possam representar um terço a metade dos MMC nos países desenvolvidos. ${ }^{14}$

$\mathrm{Na}$ literatura encontra-se descrita uma tendência para aumento tanto dos MMC in situ como dos invasivos, sendo que os casos in situ têm aumentado numa taxa superior aos invasivos, provavelmente por deteção mais precoce. ${ }^{15,16}$ Além disso, o aumento da incidência dos MMC invasivos, parece dever-se à incidência crescente dos MMC finos, com estabilização dos espessos, o que não se verificou na nossa população. ${ }^{17} \mathrm{Na}$ verdade, no que concerne ao índice de Breslow, quando comparado com o estudo prévio, verificou-

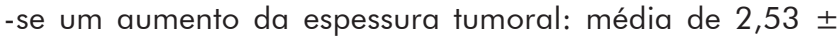
$2,62 \mathrm{~mm}$ (versus 3,01 $\pm 3,59$ ).

\section{CONCLUSÃO}

Tem-se assistido a um aumento de deteção de MMC in situ, devido à implementação de rastreios mais eficazes e ao desenvolvimento de novas tecnologias, como dermatoscopia digital e microscopia confocal, ${ }^{18}$ facto também suportado pela obtenção de um maior número de casos em comparação com o estudo prévio mais alargado (11 anos).

Apesar deste investimento, o aumento do número de diagnósticos não parece contrabalançar o risco global de $M M C$, uma vez que continuam a surgir muitos casos de MMC, predominantemente invasivos e espessos. Importa assim rever estratégias, de forma a aumentar a consciencialização das populações para os fatores de risco associados a esta neoplasia cutânea.

Conflitos de interesse: Os autores declaram não possuir conflitos de interesse.

Suporte financeiro: $O$ presente trabalho não foi suportado por nenhum subsídio ou bolsa.

Confidencialidade dos dados: Os autores declaram ter seguido os protocolos do seu centro de trabalho acerca da publicação dos dados de doentes.

Conflicts of interest: The authors have no conflicts of interest to declare.

Financing Support: This work has not received any contribution, grant or scholarship.

Confidentiality of data: The authors declare that they have followed the protocols of their work center on the publication of data from patients.

Proveniência e revisão por pares: Não comissionado; revisão externa por pares

Provenance and peer review: Not commissioned; externally peer reviewed

\section{REFERÊNCIAS}

1. Jemal A, Siegel R, Ward E, HAo Y, Xu J, Thun MJ. Cancer statistics, 2009. CA Cancer J Clin. 2009;59:225-49.

2. Duarte AF, Sousa-Pinto B, Freitas A, Delgado L, Costa-Pereira A, Correia O. Skin cancer healthcare impact: A 


\section{Artigo Original}

nation-wide assessment of an administrative database. Cancer Epidemiol. 2018t;56:154-60. doi: 10.1016/j. canep.2018.08.004.

3. Parkin DM, Bray F, Ferlay J, Pisani P. Estimating the world cancer burden: Globocan 2000. Int J Cancer. 2001; 94:153-6.

4. American Cancer Society. Cancer Facts \& Figures 2019. Atlanta: American Cancer Society; 2019.

5. Forsea AM, Del Marmol V, de Vries E, Bailey E, Geller AC. Melanoma incidence and mortality in Europe. $\mathrm{Br} \mathrm{J}$ Dermatol. 2012; 167:1124-30. doi: 10.1111/i.1365$-2133.2012 .11125 . x$

6. Ferreira M, Costa V, Torres T, Selores M. Análise retrospectiva de melanoma cutâneo primário: 1996-2006. Trab Soc Port Dermatol Venereol. 2007; 65:509-18.

7. Ríos L, Nagore E, López JL, Redondo P, Martí RM, Fernández-de-Misa R, et al. Melanoma characteristics at diagnosis from the Spanish National Cutaneous Melanoma Registry: 15 years of experience. Actas Dermosifiliogr. 2013; 104:789-99. doi: 10.1016/i.ad.2013.02.003.

8. Ferrari Júnior NM, Muller H, Ribeiro M, Maia M, Saches Júnior JA. Cutaneous melanoma: descriptive epidemiological study. São Paulo Med J.2008; 126:41-7.

9. Chevalier V, Barbe C, Le Clainche A, Arnoult G, Bernard $\mathrm{P}$, Hibon E, Grange F. Comparison of anatomical locations of cutaneous melanoma in men and women: a population-based study in France. Br J Dermatol. 2014; 171:595-601. doi: 10.1111/bid.13052.

10. Nielsen K, Måsbäck A, Olsson H, Ingvar C. A prospective, population-based study of 40,000 women regarding host factors, UV exposure and sunbed use in relation to risk and anatomic site of cutaneous melanoma. Int J Cancer.
2012; 131:706-15. doi: 10.1002/ijc.26408.

11. Lesage C, Barbe C, Le Clainche A Lesage FX, Bernard P, Grange F. Sex-related location of head and neck melanoma strongly argues for a major role of sun exposure in cars and photoprotection by hair. J Invest Dermatol. 2013; 133:1205-11. doi: 10.1038/jid.2012.405.

12. Day TA, Hornig JD, Sharma AK, Brescia F, Gillespie MB, Lathers D. Melanoma of the head and neck. Curr Treat Options Oncol. 2005; 6:19-30.

13. Rigual NR, Popat SR, Jayaprakash V, Jaggernauth W, Wong $M$. Cutaneous head and neck melanoma: the old and the new. Expert Rev Anticancer Ther. 2008;8:403-12. doi: 10.1586/14737140.8.3.403.

14. Dabouz F, Barbe C, Lesage C, Le Clainche A, Arnoult G, Hibon $E$, et al. Clinical and histological features of head and neck melanoma: a population-based study in France. $\mathrm{Br} J$ Dermatol. 2015; 172:707-15. doi: 10.1111/ bjd. 13489.

15. Curchin DJ, Harris VR, McCormack CJ, Smith SD. Changing trends in the incidence of invasive melanoma in Victoria, 1985-2015. Med J Aust. 2018; 208:265-69.

16. Wallingford SC, Alston RD, Birch JM, Green AC. Increases in invasive melanoma in England, 1979-2006, by anatomical site. Br J Dermatol. 2011 ; 165:859-64. doi: 10.1111/i.1365-2133.2011.10434.x.

17. Sacchetto L, Zanetti R, Comber H, Bouchardy C, Brewster $\mathrm{DH}$, et al. Trends in incidence of thick, thin and in situ melanoma in Europe. Eur J Cancer. 2018; 92:108-18. doi: 10.1016/i.ejca.2017.12.024.

18. Moreira J, Moreira E, Azevedo F, Mota A. Cutaneous malignant melanoma: a retrospective study of seven years (2006-2012). Acta Med Port. 2014;27:480-8. 